\title{
EVALUATION OF SCATTER FRACTION (SF) OF A 16-RING POSITRON EMISSION TOMOGRAPHY (PET) SYSTEM
}

\author{
M Monjur Ahasan \\ Atomic Energy Centre, Ramna, Dhaka, Bangladesh \\ Email: monjur_ahasan@yahoo.co.uk
}

\begin{abstract}
Scatter fraction (SF) characteristic of a newly constructed 16-ring PET system with a FOV of $20 \mathrm{~cm}$ was experimentally evaluated in 2D acquisition mode (ring difference $\leq 3$ ) but without septa, using a $10 \mathrm{~cm}$ diameter phantom. The scatter fraction measured from the backprojected images and the sinogram data aiming to find the discrepancy between the two approaches and to test the camera performance operating without septa. The images were reconstructed by the filtered backprojection (FBP) technique using some different filter functions. SF measured from the reconstructed images agreed within uncertainties and had a mean value of $26.1 \pm 0.8 \%$ at $250-850 \mathrm{keV}$ energy and $12 \mathrm{~ns}$ coincidence time window. Scatter fraction also measured from analysis of the PET sinogram was slightly lower, at 23.6 $\pm 0.7 \%$. In each case, the procedure used for estimating the background under the peak is only approximate, and this may account for the slight discrepancy between the two approaches. The results so far indicate that the system can be operated in 2D mode without septa.
\end{abstract}

Keywords: Scatter Fraction, PET, LOR, septa

\section{INTRODUCTION}

Scatter Fraction (SF) is one of the performance parameters of PET-CT imaging technology. Detection of compton scattered events is a major source of background in PET, especially when imaging objects without any collimating septa [1].

When one or both photons undergo compton interaction within the body before hitting the detectors, the event is known as a scattered event. In practice, most scattered photons are spread out of the field of view and are never detected. Those annihilations for which one or both gamma rays are scattered, but still detected, are referred to as scattered events [2]. The line-of-response (LOR) assigned to the event is uncorrelated with the original annihilation event, i.e., an incorrect LOR is formed because the photons' paths are not collinear. The contribution of scattered events is described by [Eq.1].

$$
\mathrm{S} \propto \frac{\mathrm{Z}^{3}}{\mathrm{~L} \times \mathrm{D}}
$$

Where $\mathrm{Z}$ is the axial length of the active acquisition volume, $\mathrm{D}$ is the scanner diameter, and $\mathrm{L}$ is the length of the septa. Septa are rings of lead or tungsten material, used to separate each crystal ring when the PET system is operated in 2D acquisition mode [3]. As the event is incorrectly positioned on the PET detectors, hence the effect is to add a broad background to the image. This causes errors in the radiotracer concentration by misplacing events during reconstruction. Therefore, scattered coincidences degrade both image quality (due to loss of contrast) and quantitative accuracy.

Scatter in PET can arise from three major sources: namely inside the object, detector itself, and the gantry and surrounding environment [4]. This also depends on other factors such as patient's size, density, acceptance angle, energy discriminator settings, radiotracer distribution, etc. Scattered events can be reduced by using inter-plane septa and also applying a simple energy threshold. 
The objective of the study was to evaluate the discrepancy in the analysis of scatter fraction measured from the backprojected images and the sinogram data. Also, this work was an important part in order to test the new PET camera accounting scatter fraction to get optimum image quality.

\section{MATERIALS \& METHODS}

The PET camera with a FOV of $20 \mathrm{~cm}$ was designed with 2 bucket rings each having 8 BGO detector modules. The system operated in $2 \mathrm{D}$ acquisition mode (ring difference $\leq 3$ ) but without septa, and detector modules were supported on a horizontal table as no gantry was made. Septa and rod sources like in the standard system were also not included. The ECAT [5] software was simplified to control data acquisition directly.

\subsection{Data acquisition}

All data were acquired from the PET system in 2D mode (ring difference $\leq 3$ ) but without septa. The lower and upper energy discriminator levels were set at 250 and $850 \mathrm{keV}$, respectively. The width of the coincidence time window was set at $12 \mathrm{~ns}$. The images were reconstructed by the filtered backprojection (FBP) technique using some different filter functions.

Standards were developed [6] for the measurement of SF in clinical PET scanners. In the measurement, a water filled cylindrical phantom ( $21.5 \mathrm{~cm}$ long and $10 \mathrm{~cm}$ diameter) was used. A 68-Ge line source (14.1 $\mathrm{cm}$ long and $3 \mathrm{~mm}$ diameter) with an activity of $0.37 \pm 0.04 \mathrm{MBq}$ was positioned approximately at the central axis of the phantom as in Figure 1. Coincidence events were acquired for $300 \mathrm{sec}$.

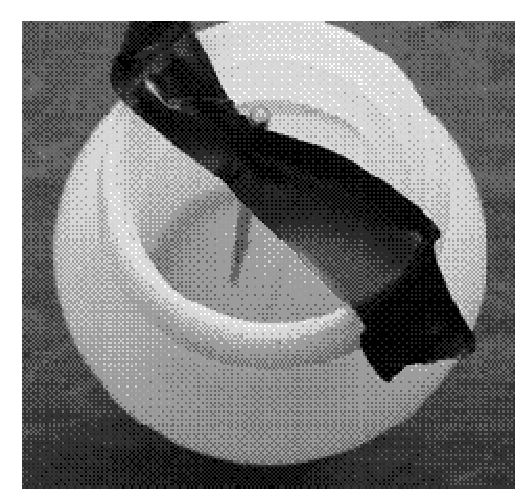

Fig.1: Experimental setup within the camera FOV for the measurement of scatter fraction (SF)

\subsection{Data reconstruction and analysis}

After acquiring the coincidence events, the sinogram data were then compressed by the scan-compress technique and normalized [7,8] by creating a normalization file "jun20cyl2.nrm". The images were reconstructed by the filtered backprojection (FBP) technique with a Hamming filter of cut-off frequency 0.4 and $256 \times 256$ matrixes with a pixel size equal to $1.9 \mathrm{~mm}$. 31 image planes were produced in each acquisition as the scanner was a 16-ring tomograph and operated in 2D mode. Among the 31 image planes, only three planes 15, 16 and 17 were considered for study. Pixel counts and the number of pixels were measured directly by selecting regions of interest (ROI) on the reconstructed images [Fig. 2]. Three concentric circular regions, $\mathrm{ROI}(1), \mathrm{ROI}(2)$ and $\mathrm{ROI}(3)$, were used, centred on the line source. Total events $I_{1}, I_{2}$ and $I_{3}$ within each region were measured by multiplying the average pixel value by the number of pixels within the region. ROI(1) had radius $10 \mathrm{~mm}$ : the true counts were assumed to be entirely contained within this ROI. ROI(2) had radius $20 \mathrm{~mm}$. The background contribution to ROI(1) was estimated using the average pixel contents in the annular region $\mathrm{ROI}(2)-\mathrm{ROI}(1)$, making the assumption 
that the scatter background is approximately flat over this central region. Then the true counts $\mathrm{T}$ are given by $\mathrm{T}=\mathrm{I}_{1}-\left(\mathrm{I}_{2}-\mathrm{I}_{1}\right) /(\mathrm{k}-1)=\left[\mathrm{kI} \mathrm{I}_{1}-\mathrm{I}_{2}\right] /(\mathrm{k}-1)$ where $\mathrm{k}$ is the ratio of areas of $\mathrm{ROI}(2)$ to $\mathrm{ROI}(1)$. Finally the scatter counts $S$ were obtained by subtracting the true counts $T$ from the total counts $I_{3}$ in $\operatorname{ROI}(3)$ and the scattered fraction was finally calculated using the well known formula:

$$
\mathrm{SF}=\frac{\mathrm{S}}{\mathrm{T}+\mathrm{S}}
$$

The scatter fraction was also measured directly from the sinogram data.

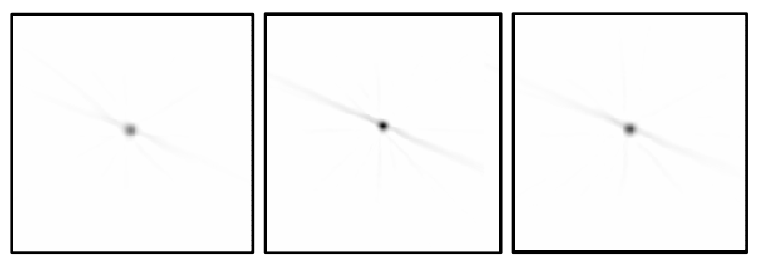

Fig. 2: Filtered backprojected PET images for a 68-Ge line source positioned at the central axis of the inactive water filled phantom (10 $\mathrm{cm}$ diameter); the images were used for the analysis of scatter fraction. Left image: plane 15 (direct plane), Middle Image: plane 16 (cross plane), Right image: plane 17 (direct plane)

\section{RESULTS}

\subsection{Image data analysis}

SF was calculated from the file named as June26line1Hm.4_256.img.

i) Image plane 15 (Direct plane):

$I_{1}=7939$ counts, $I_{2}=9523$ counts, $I_{3}=9887$ counts, $k=3.92$,

so $\mathrm{T}=[3.92 \times 7939-9523] / 2.92=7397$

The uncertainty on $\mathrm{T}$ can be derived from the Poisson uncertainties on $\mathrm{I}_{1}$ and $\mathrm{I}_{2}$ as

$$
\begin{aligned}
& \Delta T=\frac{1}{k-1} \sqrt{\left(k \Delta I_{1}\right)^{2}+\left(\Delta I_{2}\right)^{2}}=124 \\
& \mathrm{~S}=\mathrm{I}_{3}-\mathrm{T}=9887-7397=2490 \\
& \Delta \mathrm{S}=\sqrt{\left(\Delta I_{3}\right)^{2}+(\Delta T)^{2}}=159
\end{aligned}
$$

So $\mathrm{SF}=2490 / 9887=0.252$

To calculate the uncertainty on SF, note that $S F=\frac{S}{S+T}=1-\frac{T}{S+T}$,

so that the error on $\mathrm{SF}$ is equal to the error on $\frac{T}{S+T}$, 
which is $\sqrt{\left(\frac{\Delta T}{T}\right)^{2}+\left(\frac{\Delta I_{3}}{I_{3}}\right)^{2}} \frac{T}{S+T}$, giving an uncertainty 0.013

So the final result is $\mathrm{SF}=0.252 \pm 0.013$

ii) Image plane 16 (Cross plane):

$I_{1}=9844$ counts, $I_{2}=11878$ counts, $I_{3}=12441$ counts, $k=3.92$,

Using the same procedure as above, $\mathrm{T}=9147 \pm 138$

$\mathrm{S}=3294$

$\mathrm{SF}=0.265 \pm 0.013$

iii) Image plane 17 (Direct plane):

$I_{1}=7404$ counts, $I_{2}=8872$ counts, $I_{3}=9416$ counts, $k=3.92$,

Using the same procedure as above, $\mathrm{T}=6901 \pm 120$

$\mathrm{S}=2515$

$\mathrm{SF}=0.267 \pm 0.015$

Scatter fraction $(\mathrm{SF})$ and its uncertainty:

$\begin{array}{cccc}\text { Plane number } & \text { SF } & \text { Uncertainty on SF } & \text { SF (Mean) } \\ \text { Plane 15 } & 25.2 \% & 1.3 \% & \\ \text { Plane 16 } & 26.5 \% & 1.3 \% & \\ \text { Plane 17 } & 26.7 \% & 1.5 \% & 26.1 \pm 0.8 \%\end{array}$

\subsection{Sinogram data analysis}

Also, a value of SF was extracted directly from the profile (Fig. 3) across the central sinogram obtained from the same phantom. The peak was assumed to be 10 bins wide, and the background under the peak was estimated using the average counts in the 5 bins immediately below the peak and the 5 bins immediately above. This analysis resulted in a value of $\mathrm{SF}=0.236 \pm 0.007$.

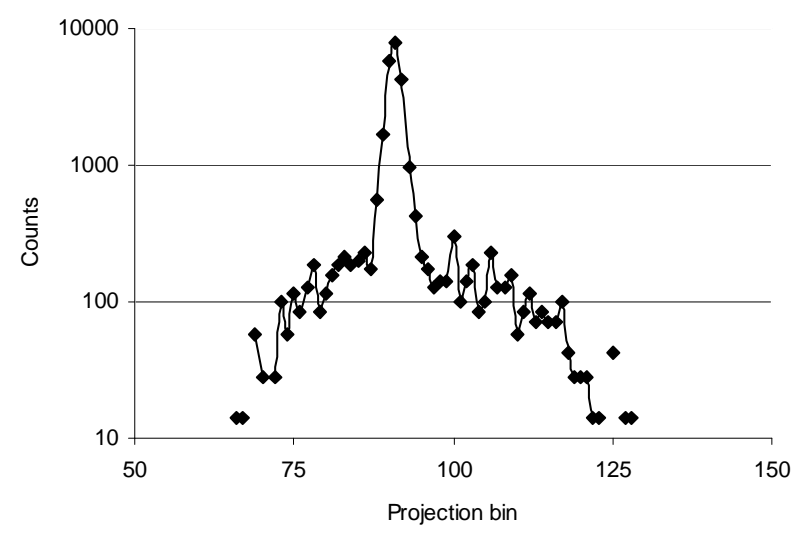

Fig. 3: Profile across the sinogram measured for a $68-\mathrm{Ge}$ line source at the centre of the $10 \mathrm{~cm}$ diameter cylinder of water 


\section{DISCUSSION}

Scatter fraction is a measure of the fraction of total (un-scattered and scattered) events in the acquired PET data, excluding randoms, which have scattered prior to detection. This fraction is most commonly measured as part of the count rate performance study [9] of a PET system under the assumption of lowactivity data containing negligible randoms. The process of SF measurement is performed by acquiring only prompt events, which includes un-scattered (true), scattered and randoms/delayed events.

The values of SF obtained from the backprojected images agreed within uncertainties and had a mean value of $26.1 \pm 0.8 \%$. The value of SF obtained from analysis of the sinogram was slightly lower, at 23.6 $\pm 0.7 \%$. In each case, the procedure used for estimating the background under the peak is only approximate, and this may account for the slight discrepancy between the two approaches.

Another study [10] also measured SF using a line source inside a $10 \mathrm{~cm}$ diameter water fraction, and obtained values of $18.5 \%$ (high sensitivity $2 \mathrm{D}$ mode) and $29.8 \%$ (3D mode). As expected, the experimental results were intermediate between these values, since miniPET is operated in 2D acquisition mode (ring difference $\leq 3$ ) but without septa.

\section{CONCLUSION}

Scatter fraction measured from the filtered backprojected images agreed within uncertainties. The value of SF obtained from analysis of the sinogram was slightly lower. In each case, the procedure used for estimating the background under the peak is only approximate, and this may account for the slight discrepancy between the two approaches. As expected, the results were intermediate between these values, since the PET camera was operated in 2D mode but without inter-plane septa in order to get optimum image quality.

\section{REFERENCES}

1. R. E. Schmitz, A. M. Alessio and P. E. Kinahan, 2005. The physics of PET/CT scanners in "PET and PET/CT: A clinical guide", $2^{\text {nd }}$ Ed, E. Lin and A. Alavi, Thieme Ch 1: 3-14

2. J. M. Ollinger and J. A. Fessler, 1997. Positron emission tomography. IEEE Signal Processing Magazine; 14(1):43-55.

3. G. B. Shaha, 2010. Basics of PET Imaging: Physics, Chemistry, and Regulations, Springer, $2^{\text {nd }}$ Ed, Ch 3:4168

4. Y. Yang and S. R. Cherry 2006. Observations regarding scatter fraction and NEC measurements for small animal PET. IEEE Trans. Nucl. Sci. 53(1):127-132.

5. ECAT 951, 953 Service Manual. Siemens Medical System, Testing \& Diagnostics, 1985.

6. NEMA Standards Publication NU2-2001: Performance measurement of positron emission tomographs, National Electrical Manufactures Association, Rosslyn, VA, 2001.

7. M. M. Ahasan and D. J. Parker, 2008. Initial results from a prototype large ring PET scanner. In: L. Gelman, D. W. Hukins, A. Hunter, A. M. Korsunsky editors. Proceedings of the World Congress on Engineering 2008. London: Lecture Notes in Engineering and Computer Science, 1: 676-681.

8. M. M. Ahasan and D. J. Parker, 2009. Design and initial performance evaluation of a prototype large ring PET scanner in "Advances in Electrical Engineering and Computational Science", S I Ao et al (Eds) Springer, 14: $161-172$. 
9. S. C. Strother, M. E. Casey and E. J. Hoffmann 1990. Measuring PET scanner sensitivity: relating count rates to image signal-to-noise ratios using noise equivalent counts. IEEE Trans. Nucl. Sci. 37:783-788.

10. M. Watanabe, H. Okada, K. Shimizu, T. Ohmura, E. Yoshikawa, T. Kosugi, S. Mori and T. Yamashita 1996. A high resolution animal PET scanner using compact PS-PMT detectors. Nucl. Sci. Symp (Conference Record IEEE). 2:1330-1334. 